\title{
BMJ Open Lorcaserin for prevention and remission of type 2 diabetes mellitus in people with overweight or obesity: protocol for a systematic review and meta-analysis
}

\author{
Hai Zeng, ${ }^{1}$ Meng Luo, ${ }^{2}$ Zunjiang Li, ${ }^{2}$ Junru Wen, ${ }^{3,4}$ Guoxin He, ${ }^{5}$ Yuelin Jin, ${ }^{4}$ \\ Wenbin Fu, ${ }^{6,7}$ Peng Zhou ${ }^{8}$
}

To cite: Zeng H, Luo M, Li Z, et al. Lorcaserin for prevention and remission of type 2 diabetes mellitus in people with overweight or obesity: protocol for a systematic review and meta-analysis. BMJ Open 2019;9:e029426. doi:10.1136/ bmjopen-2019-029426

- Prepublication history and additional material for this paper are available online. To view these files, please visit the journal online (http://dx.doi org/10.1136/bmjopen-2019029426).

$\mathrm{HZ}$, ML and ZL contributed equally.

Received 28 January 2019 Revised 23 April 2019 Accepted 11 June 2019

\section{ABSTRACT}

Introduction High body mass index (BMI) is associated with risk of diabetes. Lorcaserin is a selective 5-hydroxytryptamine $2 \mathrm{C}$ agonist which exerts robust benefits on long-term weight loss by suppressing appetite among adults with overweight or obesity. The magnitude of efficacy of lorcaserin for preventing and remitting type 2 diabetes mellitus (T2DM) among those people remains undefined. Therefore, we plan to conduct this systematic review and meta-analysis to aggregate data from all published studies with regard to the issue to acquire reliable evidence.

Methods and analysis We will search various databases for relevant trials published up to June 2019. Randomised controlled trials investigating the efficacy of lorcaserin for preventing and remitting T2DM among overweight and obese population will be included. A standardised data form will be used to complete data search and extraction in duplicate. All discrepancies will be resolved by consensus. The primary outcome will be incidence of T2DM in patients with pre-diabetes. Secondary outcomes will include achievement of normoglycaemia in people with pre-diabetes, remission of hyperglycaemia in patients with diabetes, the proportion of patients with weight loss of at least $5 \%$ or $10 \%$ and hypoglycaemia incident. Data synthesis and statistical analysis will be performed for each outcome with Stata V.14.0.

Ethics and dissemination Ethics approval is not required. Results of our study will be submitted to a peerreview journal.

PROSPERO registration number CRD42019119136.

\section{INTRODUCTION}

Over the past 40 years, the global prevalence of obesity has reached alarming proportions with rates nearly tripling. ${ }^{1}$ As of 2016 , $39 \%$ of adults all over the world were overweight (defined as body mass index (BMI) $\left.25-29.9 \mathrm{~kg} / \mathrm{m}^{2}\right)$, and another $13 \%$ of adults were obese $\left(\mathrm{BMI} \geq 30 \mathrm{~kg} / \mathrm{m}^{2}\right)$. ${ }^{1}$ Obesity has been linked to the development and progression of dysglycaemia and type 2 diabetes mellitus (T2DM). ${ }^{2}$ Analogously, the prevalence of diabetes is also rising globally,
Strengths and limitations of this study

- This study is the first systematic review and meta-analysis combining data from all available randomised controlled trials to evaluate the effectiveness of lorcaserin on diabetes prevention and remission among people with overweight or obesity.

- The study design is rigorous and complete, including systematic literature search, comprehensive evaluation of clinically important outcomes and proper assessment of quality of evidence.

- The quantity, methodological quality and heterogeneity of included studies may influence the results of this study.

- This meta-analytic review is limited by the inability to use individual participant data.

the number of people with diabetes grew from 153 million in 1980 to as many as 476 million in 2017, with T2DM accounting for 463 million. ${ }^{34}$ Approximately 29 million adults in America had T2DM in 2012, which is projected to increase to 100 million before $2050 .{ }^{56}$ In addition, an estimated 280 million adults globally, including 57 million in America, are considered to be patients with pre-diabetes. ${ }^{7}$ Pre-diabetes is an umbrella term, describing a blood glucose level that lies above normal but below that diagnosed for T2DM. ${ }^{9}$ People with defined pre-diabetic state are considered to be at high risk for the development of T2DM. ${ }^{10-12}$ Both diabetes and obesity raise the risk of cardiovascular disease, chronic kidney disease, neuropathy, stroke and cancers. ${ }^{13-20}$ The two conditions are also related to increased risk of all-cause mortality, the mortality burden of which nearly doubled in the past three decades. ${ }^{1321}$ Globally, 6.53 million deaths and 171 million disability-adjusted life-years (DALYs) were attributed to high fasting plasma glucose in 2017, followed by high BMI (4.72 million 
deaths and 148 million DALYs). ${ }^{22}$ Pharmacological agents for weight loss are recommended in guidelines as adjuncts to comprehensive lifestyle interventions in long-term weight management, which exert benefits on preventing progression to pre-diabetes and diabetes, reducing hypertension risk and improving lipid profiles, ${ }^{13}{ }^{23}$ especially for those persons who fail in achieving sufficient weight loss with lifestyle interventions alone. ${ }^{24}$

As a highly selective serotonin $2 \mathrm{C}$ receptor agonist on proopiomelanocortin neurons, lorcaserin is effective for weight loss by regulating appetite in adults with obesity. ${ }^{25}$ Based on data by three main clinical trials, including behavioural modification and lorcaserin for overweight and obesity management (BLOOM) study, BLOOM in patients of diabetes mellitus study and behavioural modification and lorcaserin second study for obesity management study, the US Food and Drug Administration initially approved lorcaserin as a new adjunctive prescription drug to lifestyle modification (reduced-calorie diet and increased physical activity) for the treatment of obesity in 2012, bringing new hope for people with obesity. ${ }^{25-27}$ Lorcaserin, considered as a safe and well tolerable drug, could achieve statistically significant weight loss and improve cardiometabolic parameters. ${ }^{28}$ Additionally, long-term data from a recent large randomised trial suggested that lorcaserin could reduce the risk of microvascular complications without an increased incidence of major adverse cardiovascular events. ${ }^{29} 30$ Thus, lorcaserin demonstrates superiority to several pharmacological weight-loss agents associated with various cardiovascular or neuropsychiatric complications. ${ }^{31-33}$

In recent years, there is an increasing interest regarding the prevention of progression of pre-diabetes to diabetes and remission of hyperglycaemia, and meanwhile lorcaserin has now been investigated in several trials ${ }^{29} 34-36$ in patients with type 2 diabetes or pre-diabetes and obesity. Results from these trials suggest that lorcaserin could help with achieving clinically meaningful weight loss, promoting reversion to euglycaemia and preventing progression to T2DM in patients with obesity. ${ }^{29}{ }^{34-36}$ However, there is a paucity of comprehensive evidence of randomised controlled trials (RCTs) with respect to this topic. Our meta-analytic review aims to combine data from all relevant RCTs of lorcaserin to acquire reliable estimates regarding specific metabolic efficacy and safety outcomes on preventing and remitting T2DM in patients with obesity.

\section{METHODS}

\section{Study design}

This protocol is reported in line with the Preferred Reporting Items for Systematic Review and Meta-Analysis Protocols (PRISMA-P) guidelines. ${ }^{37}{ }^{38}$ This study will be conducted according to the Preferred Reporting Items for Systematic Reviews and Meta-Analyses (PRISMA) statements. $^{39}$

\section{Study registration}

According to PRISMA-P and PRISMA guidelines, this study has been registered in the International Prospective Register of Systematic Reviews (PROSPERO). We anticipate to conduct this review from January to June 2019.

\section{Eligibility criteria}

Population

Eligible patients will be adults (older than 18 years) who have T2DM or pre-diabetes with coexisting obesity. Definitions of pre-diabetes and diabetes could be based on any recognised standard diagnosis criteria, for instance, the American Diabetes Association guidelines.

\section{Interventions and comparators}

We plan to investigate the comparisons of lorcaserin versus placebo.

\section{Outcomes}

The primary outcome will be incidence of T2DM in patients with pre-diabetes. Secondary outcomes will include achievement of normoglycaemia among pre-diabetic patients, remission of hyperglycaemia in patients with diabetes, the proportion of patients who lost $\geq 5 \%$ or $10 \%$ body weight among people with pre-diabetes or diabetes and incidence of hypoglycaemia in patients with diabetes. Definitions for these outcomes should be established and described in eligible trials.

\section{Type of studies}

RCTs will be included in our study. Retrospective or prospective observational cohort studies, cross-section studies, case-control studies and reviews will be excluded.

\section{Search strategy}

We will conduct a comprehensive systematic search of the following databases from inception date to June 2019, including PubMed, Embase, Web of Science, the Cochrane library, ClinicalTrials.gov and the WHO International Clinical Trials Registry Platform. Moreover, a restriction on the language of publication to English will be placed. Relevant keywords and their combinations will be applied in the search strategy, for instance, diabetes, T2DM, pre-diabetes, pre-diabetic state, glucose intolerance, impaired glucose, diabetic, dysglycaemia, hyperglycaemia, lorcaserin, obesity, obese, overweight, conversion, delay and prevent. A complete search strategy of PubMed database is provided in online supplementary. All potentially eligible studies will be considered for review. In addition, using the reference lists of key articles, including retrieved included studies, meta-analyses and systematic reviews, we will also do a manual search for other relevant eligible trials.

\section{Selection of studies}

According to the predefined inclusion criteria, titles and abstracts of all records retrieved in the literature search will be scanned by two review authors. Trials that met the inclusion criteria will be retrieved for further assessment. 
All potentially relevant articles will be investigated via scrutinising full text. The PRISMA flow diagram of study selection will be used to depict trial identification and exclusion process. Disagreements at study selection will be resolved by discussion and consensus.

\section{Data collection process}

Two review authors will independently extract data of all eligible studies with the use of a standardised data form in duplicate. The following data will be extracted from all selected studies: trial characteristics (author, year of publication, country setting, study design and trial duration), participant characteristics (mean age, percent of male, race, baseline weight, BMI and glycemic parameters), dose and frequency of lorcaserin and predefined primary and secondary outcomes. Any disagreement will be resolved by consensus or recourse to a third reviewer. We will contact authors by e-mails to acquire more information if the data are insufficient in the original article.

\section{Assessment of methodological quality}

The Cochrane tool for assessing risk of bias will be used to evaluate the methodological quality of included RCTs by two review authors working independently. The assessment details include seven domains as follows: random sequence generation, allocation concealment, blinding of participants and personnel, blinding of outcome assessment, incomplete outcome data, selective reporting and other bias. ${ }^{40}$ Based on the description details of eligible studies, each domain will be defined as being at high, low or unclear risk of bias. We will settle all discrepancies in quality evaluation through consensus and consultation with a third reviewer.

\section{Data synthesis and statistical analysis}

Stata V.14.0 (StataCorp) will be used to carry out all statistical analyses. We will pool mean differences with 95\% CIs for the effect of continuous outcomes across trials. The overall estimates of the risk ratios and their associated $95 \%$ CIs will also be calculated for categorical outcomes. A p value of no more than 0.05 will be considered as statistically significant. Results will be synthesised by a random-effects model to adequately account for the heterogeneity among included studies. The heterogeneity across included trials will be assessed by $\mathrm{I}^{2}$ statistic. Heterogeneity will be considered to be low $\left(\mathrm{I}^{2}<25 \%\right)$, moderate $\left(\mathrm{I}^{2}=25 \%-50 \%\right)$ or high $\left(\mathrm{I}^{2}>75 \%\right) .{ }^{41}$ Potential publication bias will be evaluated by funnel plots, and also assessed by the Egger and Begg tests if no less than 10 studies are pooled. ${ }^{42}$

\section{Sensitivity and subgroup analyses}

A sensitivity analysis will be conducted by excluding studies with high risk of bias. In addition, the leaveone-out sensitivity analysis will also be performed to evaluate the stability of results. Moreover, subgroup analysis will be conducted according to BMI $\left(25-29.9 \mathrm{~kg} / \mathrm{m}^{2}\right.$ and $\geq 30 \mathrm{~kg} / \mathrm{m}^{2}$ ). Outcome data on adults (18-64 years) and elderly population (aged 65 years or older) will be analysed separately. Gender and ethnicity will also be considered for subgroup analyses if the data permit.

\section{Quality of evidence}

The grading of recommendations assessment, development and evaluation (GRADE) approach will be applied to rate the overall quality of evidence with respect to each outcome of our findings. ${ }^{43}$ The GRADE method categorises the quality of evidence into four levels as follows: very low, low, moderate and high. Several reasons will influence the confidence of evidence, including reporting bias, study limitations, indirectness of evidence, inconsistency of results and imprecision.

\section{Patient and public involvement}

Patients and public will be not involved in the study.

\section{Ethics and dissemination}

The meta-analytic review will not analyse confidential patient data, thus formal ethics approval is not required. Our results will be reported in line with the framework laid out in the PRISMA guidelines. The findings from our study will be submitted to a peer-reviewed journal.

\section{DISCUSSION}

Over the recent years, lorcaserin has gained increasing popularity in weight management among patients with obesity, with additional evidence from trials suggesting proven benefits on preventing progression of pre-diabetes to T2DM and remission of hyperglycaemia. Our study will be the first systematic review and meta-analysis to assess effectiveness and safety of lorcaserin for preventing and remitting of type 2 diabetes in the population with adiposity. The conclusions of this study are of benefit to patients with coexisting pre-diabetes or T2DM and obesity, guideline developers and clinicians. Nevertheless, we should acknowledge several potential limitations of this study. Trial-level data will be aggregated instead of individual participant data. Moreover, differences in inclusion criteria, definitions of endpoints and methodological quality among included studies may cause considerable heterogeneity. Additionally, common to any meta-analysis, the quantity of included studies may influence the findings of this study. To include more relevant studies and provide more reliable evidence, we will conduct a comprehensive systematic search of various databases. In the case of limited number of available trials, we will present a narrative review simultaneously and interpret the findings of our study cautiously.

\section{Author affiliations}

${ }^{1}$ Department of Acupuncture and Moxibustion, The Second Clinical College,

Guangzhou University of Chinese Medicine, Guangzhou, China

${ }^{2}$ The Second Clinical College, Guangzhou University of Chinese Medicine, Guangzhou, China

${ }^{3}$ Shanghai University of Traditional Chinese Medicine, Shanghai, China ${ }^{4}$ Shanghai University of Medicine \& Health Sciences, Shanghai, China

${ }^{5}$ The First Clinical College, Guangzhou University of Chinese Medicine, Guangzhou, China 
${ }^{6}$ Department of Acupuncture and Moxibustion, Guangdong Provincial Hospital of Chinese Medicine, Guangzhou, China

${ }^{7}$ Shenzhen Bao'an Research Center for Acupuncture and Moxibustion, Shenzhen, China

${ }^{8}$ Department of Acupuncture and Moxibustion, Shenzhen Bao'an Traditional Chinese Medicine Hospital Group, Shenzhen, China

Contributors WF and YJ were the guarantors. HZ, ML and JW contributed to study design. JW, ML and ZL drafted the protocol. GH and ML registered the protocol and designed the search strategy. HZ, WF, YJ and PZ revised the protocol. All authors reviewed and approved the final manuscript.

Funding This research was supported by the Sanming Project of Medicine in Shenzhen (No. SZSM201806077) and Shenzhen Bao'an Traditional Chinese Medicine Hospital Group.

Competing interests None declared.

Patient consent for publication Not required

Provenance and peer review Not commissioned; externally peer reviewed.

Open access This is an open access article distributed in accordance with the Creative Commons Attribution Non Commercial (CC BY-NC 4.0) license, which permits others to distribute, remix, adapt, build upon this work non-commercially, and license their derivative works on different terms, provided the original work is properly cited, appropriate credit is given, any changes made indicated, and the use is non-commercial. See: http://creativecommons.org/licenses/by-nc/4.0/.

\section{REFERENCES}

1. WHO. Obesity and overweight fact sheet. Geneva: World Health Organization, 2016.

2. Bray GA, Heisel WE, Afshin A, et al. The Science of Obesity Management: An Endocrine Society Scientific Statement. Endocr Rev 2018;39:79-132.

3. Danaei G, Finucane MM, Lu Y, et al. National, regional, and global trends in fasting plasma glucose and diabetes prevalence since 1980: systematic analysis of health examination surveys and epidemiological studies with 370 country-years and 2.7 million participants. Lancet 2011;378:31-40.

4. GBD 2017 Disease and Injury Incidence and Prevalence Collaborators. Global, regional, and national incidence, prevalence, and years lived with disability for 354 diseases and injuries for 195 countries and territories, 1990-2017: a systematic analysis for the Global Burden of Disease Study 2017. Lancet 2018;392:1789-858.

5. Centers for Disease Control and Prevention. National Diabetes Statistics Report Estimates of Diabetes and Its Burden in the United States. Atlanta, GA: US Department of Health and Human Services, 2014.

6. Boyle JP, Thompson TJ, Gregg EW, et al. Projection of the year 2050 burden of diabetes in the US adult population: dynamic modeling of incidence, mortality, and prediabetes prevalence. Popul Health Metr 2010;8:29.

7. Garber AJ, Handelsman Y, Einhorn D, et al. Diagnosis and management of prediabetes in the continuum of hyperglycemia: when do the risks of diabetes begin? A consensus statement from the American College of Endocrinology and the American Association of Clinical Endocrinologists. Endocr Pract 2008;14:933-46.

8. Perreault L, Temprosa M, Mather KJ, et al. Regression from prediabetes to normal glucose regulation is associated with reduction in cardiovascular risk: results from the Diabetes Prevention Program outcomes study. Diabetes Care 2014;37:2622-31.

9. Yudkin JS, Montori VM. The epidemic of pre-diabetes: the medicine and the politics. BMJ 2014;349:g4485.

10. Moelands SV, Lucassen PL, Akkermans RP, et al. Alpha-glucosidase inhibitors for prevention or delay of type 2 diabetes mellitus and its associated complications in people at increased risk of developing type 2 diabetes mellitus. Cochrane Database Syst Rev 2018;12:Cd005061.

11. American Diabetes Association. 2. Classification and Diagnosis of Diabetes: Standards of Medical Care in Diabetes-2019. Diabetes Care 2019;42:S13-28.

12. American Diabetes Association. 3. Prevention or Delay of Type 2 Diabetes: Standards of Medical Care in Diabetes-2019. Diabetes Care 2019;42:S29-33.

13. Jensen MD, Ryan DH, Apovian CM, et al. 2013 AHA/ACC/TOS guideline for the management of overweight and obesity in adults: a report of the American College of Cardiology/American Heart
Association Task Force on Practice Guidelines and The Obesity Society. Circulation 2014;129:S102-38.

14. Garofalo C, Borrelli S, Minutolo R, et al. A systematic review and meta-analysis suggests obesity predicts onset of chronic kidney disease in the general population. Kidney Int 2017;91:1224-35.

15. Singh GM, Danaei G, Farzadfar F, et al. The age-specific quantitative effects of metabolic risk factors on cardiovascular diseases and diabetes: a pooled analysis. PLoS One 2013;8:e65174.

16. Thomas G, Sehgal AR, Kashyap SR, et al. Metabolic syndrome and kidney disease: a systematic review and meta-analysis. Clin J Am Soc Nephrol 2011;6:2364-73.

17. Callaghan BC, Xia R, Reynolds E, et al. Association Between Metabolic Syndrome Components and Polyneuropathy in an Obese Population. JAMA Neurol 2016;73:1468-76.

18. Conlin PR, Colburn J, Aron D, et al. Synopsis of the 2017 U.S. Department of Veterans Affairs/U.S. Department of Defense Clinical Practice Guideline: Management of Type 2 Diabetes Mellitus. Ann Intern Med 2017;167:655-63.

19. Suh S, Kim KW. Diabetes and cancer: is diabetes causally related to cancer? Diabetes Metab J 2011;35:193-8.

20. Bhaskaran K, Douglas I, Forbes $\mathrm{H}$, et al. Body-mass index and risk of 22 specific cancers: a population-based cohort study of $5 \cdot 24$ million UK adults. Lancet 2014;384:755-65.

21. Global Burden of Metabolic Risk Factors for Chronic Diseases Collaboration. Cardiovascular disease, chronic kidney disease, and diabetes mortality burden of cardiometabolic risk factors from 1980 to 2010: a comparative risk assessment. Lancet Diabetes Endocrinol 2014;2:634-47.

22. GBD 2017 Risk Factor Collaborators. Global, regional, and national comparative risk assessment of 84 behavioural, environmental and occupational, and metabolic risks or clusters of risks for 195 countries and territories, 1990-2017: a systematic analysis for the Global Burden of Disease Study 2017. Lancet 2018;392:1923-94.

23. Garvey WT, Garber AJ, Mechanick Jl, et al. American association of clinical endocrinologists and american college of endocrinology position statement on the 2014 advanced framework for a new diagnosis of obesity as a chronic disease. Endocr Pract 2014:20:977-89.

24. Yanovski SZ, Yanovski JA. Long-term drug treatment for obesity: a systematic and clinical review. JAMA 2014;311:74-86.

25. Brashier DB, Sharma AK, Dahiya N, et al. Lorcaserin: A novel antiobesity drug. J Pharmacol Pharmacother 2014;5:175-8.

26. Redman LM, Ravussin E. Lorcaserin for the treatment of obesity. Drugs Today 2010;46:901-10.

27. Taylor JR, Dietrich E, Powell JG. New and emerging pharmacologic therapies for type 2 diabetes, dyslipidemia, and obesity. Clin Ther 2013;35:A3-A17.

28. Aronne L, Shanahan W, Fain R, et al. Safety and efficacy of lorcaserin: a combined analysis of the BLOOM and BLOSSOM trials. Postgrad Med 2014;126:7-18

29. Bohula EA, Scirica BM, Inzucchi SE, et al. Effect of lorcaserin on prevention and remission of type 2 diabetes in overweight and obese patients (CAMELLIA-TIMI 61): a randomised, placebo-controlled trial. Lancet 2018;392:2269-79.

30. Bohula EA, Wiviott SD, McGuire DK, et al. Cardiovascular Safety of Lorcaserin in Overweight or Obese Patients. N Engl J Med 2018;379:1107-17.

31. Connolly HM, Crary JL, McGoon MD, et al. Valvular heart disease associated with fenfluramine-phentermine. $N$ Engl J Med 1997:337:581-8.

32. James WP, Caterson ID, Coutinho W, et al. Effect of sibutramine on cardiovascular outcomes in overweight and obese subjects. $N$ Engl $J$ Med 2010;363:905-17.

33. Topol EJ, Bousser MG, Fox KA, et al. Rimonabant for prevention of cardiovascular events (CRESCENDO): a randomised, multicentre, placebo-controlled trial. Lancet 2010;376:517-23.

34. Magkos F, Nikonova E, Fain R, et al. Effect of lorcaserin on glycemic parameters in patients with type 2 diabetes mellitus. Obesity 2017;25:842-9

35. Nesto R, Fain R, Li Y, et al. Evaluation of lorcaserin on progression of prediabetes to type 2 diabetes and reversion to euglycemia. Postgrad Med 2016;128:364-70.

36. O'Neil PM, Smith SR, Weissman NJ, et al. Randomized placebocontrolled clinical trial of lorcaserin for weight loss in type 2 diabetes mellitus: the BLOOM-DM study. Obesity 2012;20:1426-36.

37. Moher D, Shamseer L, Clarke M, et al. Preferred reporting items for systematic review and meta-analysis protocols (PRISMA-P) 2015 statement. Syst Rev 2015;4:1.

38. Shamseer L, Moher D, Clarke M, et al. Preferred reporting items for systematic review and meta-analysis protocols (PRISMA-P) 2015 elaboration and explanation. BMJ 2015;350:g7647. 
39. Liberati A, Altman DG, Tetzlaff J, et al. The PRISMA statement for reporting systematic reviews and meta-analyses of studies that evaluate healthcare interventions: explanation and elaboration. $B M J$ 2009;339:b2700.

40. Higgins JP, Altman DG, Gøtzsche PC, et al. The Cochrane Collaboration's tool for assessing risk of bias in randomised trials. BMJ 2011;343:d5928.
41. Higgins JP, Thompson SG, Deeks JJ, et al. Measuring inconsistency in meta-analyses. BMJ 2003;327:557-60.

42. Sterne JA, Egger M, Smith GD. Systematic reviews in health care: Investigating and dealing with publication and other biases in metaanalysis. BMJ 2001;323:101-5.

43. Guyatt GH, Oxman AD, Vist GE, et al. GRADE: an emerging consensus on rating quality of evidence and strength of recommendations. BMJ 2008;336:924-6. 